\title{
The Crystal Structure of Poly(ethylene furanoate)
}

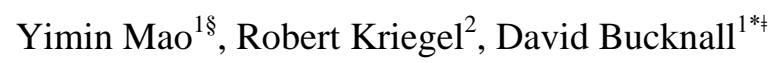 \\ 1. School of Materials Sciences and Engineering, Georgia Institute of Technology, Atlanta, Georgia \\ 30332, US \\ 2. The Coca-Cola Company, Atlanta, Georgia 30313, US \\ $\S$ Current affiliations: Department of Materials Science and Engineering, University of Maryland, College \\ Park, MD 20742 US, and NIST center for Neutron Research, National Institute of Standards and \\ Technology, Gaithersburg, MD 20899 US \\ ₹ Current affiliation: School of Engineering and Physical Sciences, Heriot-Watt University, Edinburgh \\ EH14 4AS UK \\ * Corresponding author. \\ Email: david.bucknall@hw.ac.uk
}

\begin{abstract}
The crystal structure of poly(ethylene furanoate) (PEF) has been determined using X-ray fiber diffraction (XRFD) combined with molecular modeling. Analysis of the XRFD data suggested the space group was $\mathrm{P} 2{ }_{1}$, with a monoclinic unit cell where $a=5.784 \AA$, $b=6.780 \AA, c=20.296 \AA$, and $\gamma=103.3^{\circ}$, giving a calculated unit cell density of the crystal phase was $1.562 \mathrm{~g} / \mathrm{cm}^{3}$. The repeating unit consisted of two monomers related to each other by a $2_{1}$ screw axis. It was also shown that two PEF chains were included in each unit cell, with the center-chain being staggered with respect to the edge-chain. Polymer chains were found to be near planar, although careful evaluation showed that the best fit with the data required the cell to contain a mixture of $3 / 12$ and $5 / 12$ center-chain-staggering.
\end{abstract}

(C) 2016. This manuscript version is made available under the Elsevier user license http://www.elsevier.com/open-access/userlicense/1.0/ 


\section{Introduction}

Poly(ethylene terephthalate) (PET) is widely exploited in packaging industries and for instance is prominently used for water and soft drinks bottles. However, despite being extremely widely used in such applications, PET has its limitations, particularly in respect of cost as well as issues of finite shelf life of packaged products due to its inherent barrier properties. To this end, initiatives are being explored relating both to the costs, i.e., in generating the polymer from renewable feedstocks for instance, as well as ways to improve its barrier properties. The latter includes multilayer approaches and also inclusion of additives to the PET. ${ }^{1}$ An alternative approach is the possibility of replacing PET with another polymer that possesses better barrier properties, and an obvious point to start the search is to explore other polyesters.

Poly(ethylene furanoate) (PEF) is a furan-derived analog of PET, and has the chemical structure shown in Figure 1. As can be seen, from a structural point of view, the only difference between PEF and PET is that the backbone benzene ring in the PET monomer is replaced by a furan ring in PEF. However, this difference in the primary structure leads to profound consequences in the molecular dynamics, crystal structure, as well as mechanical and barrier properties of the material. ${ }^{2-7}$ With respect to potential applications for plastic beverage bottles, PEF has been found to possess much better gas barrier properties compared to PET, with $\mathrm{O}_{2}$ and $\mathrm{CO}_{2}$ gas permeability that are 10 times and 19 times lower, respectively. ${ }^{5,7}$

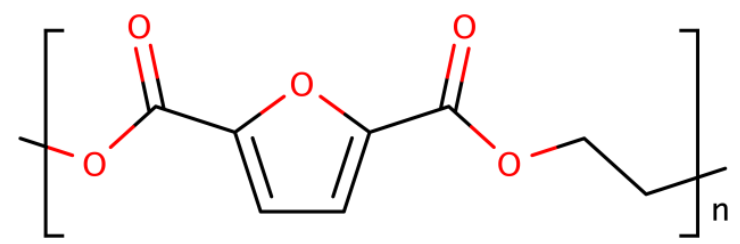

Figure 1. Chemical structure of Poly(ethylene furanoate).

The crystal structure of PET was determined more than 60 years ago, ${ }^{8}$ and consists of a triclinic unit cell with $a=4.56 \AA, b=5.94 \AA, c=10.75 \AA, \alpha=98.5^{\circ} \beta=118^{\circ}$, and $\gamma=112^{\circ}$. The repeating unit along the $c$-axis contains one monomer, with the polymer chain tilted by $\sim 5^{\circ}$ with respect to the $c$-axis. Whilst the structure of PET has been widely studied the same is not true of PEF, and only a handful of papers have been published describing its crystal structure and crystallization behavior. Part of the reason for this is that compared to other semi-crystalline polymers such as PET, PEF inherently produces solids with a very low degree of crystallinity of at most $\sim 20 \%$. In addition, the crystallization kinetics are known to be much slower than that of PET, which generates challenges in producing polymers with high enough crystallinity to characterize easily. Some general properties regarding PEF's crystallization kinetics and polymorphism have been recently reported..$^{9-11}$ 
The first, and to the best of our knowledge, the only paper on the crystal structure determination of PEF was reported by Kazaryan and Medvedeva in $1968 .{ }^{12}$ They proposed a triclinic unit cell with dimensions of $a=5.75 \AA, b=5.35 \AA, c=20.10 \AA, \alpha=133.3^{\circ}, \beta=90^{\circ}$ and $\gamma=112^{\circ}$ based on X-ray fiber diffraction (XRFD) results. These authors prepared fiber samples by first stretching the polymer at $90{ }^{\circ} \mathrm{C}$ by a factor of two, and then annealing it at $160{ }^{\circ} \mathrm{C}$ for 1 hour. In the current paper, we propose a different crystal structure based on XRFD data with improved quality. To prepare aligned crystal with higher quality, the PEF film was annealed at higher temperature for much longer time (see experimental section for details). Careful fiber tilting measurement to examine the meridian diffraction spots was carried out at synchrotron X-ray beamline using a large area detector. More diffraction spots can be resolved owing to these improvements.

Due to intrinsic properties of PEF, such as low crystallinity, small crystal size, and disorder, etc., significant broadening of the measured diffraction spots was observed, making the standard approach of using structure refinement based on the precise determination of peak position and intensity challenging. We report a method that combines crystallographic methods and molecular modeling that ultimately allow us to make direct comparison of simulated 2D diffraction patterns with experimental results, leading to solution of the complex nature of the crystal structure of PEF.

\section{Experimental Methods}

\section{Materials and sample preparation}

PEF polymer (in the form of melt extruded pellets) was provided by Coca-Cola Company. The glass transition and melting temperature of raw PEF pellet based on Differential Scanning Calorimetry (DSC) data were $86.2{ }^{\circ} \mathrm{C}$ and $219.1{ }^{\circ} \mathrm{C}$ (endothermic peak position), respectively.

The PEF polymer was dried under vacuum at $140{ }^{\circ} \mathrm{C}$ for 3 days before use to remove residual water absorbed by the polymer during storage. The polymer pellets were then melted and pressed into films of homogeneous thickness using a simple mold in a Carver hot press machine with platens set at $240{ }^{\circ} \mathrm{C}$ for 5 min, i.e., $\sim 20{ }^{\circ} \mathrm{C}$ above its melting point. After pressing, the film was removed from the hot press and allowed to cool down to room temperature. At this stage, the polymer is fully amorphous, due to its slow crystallization kinetics, as verified by both DSC and wide-angle X-ray diffraction (WAXD) measurements. The resulting films had a thickness of $0.8 \mathrm{~mm}$, and were cut into $6 \mathrm{~mm}$ x $26 \mathrm{~mm}$ rectangular samples using a fine toothed electric saw for subsequent uniaxial stretching treatment. 
A Linkam T350 tensile stage equipped with a temperature control unit was used to stretch and anneal the PEF samples. The procedure for preparing oriented crystalline samples for X-ray fiber diffraction experiments is as follows. The sample was mounted in the Linkam stage and heated to $90{ }^{\circ} \mathrm{C}$, then uniaxially stretched at a strain rate of $50 \mu \mathrm{m} / \mathrm{min}$, until the force reached $\sim 190 \mathrm{~N}$. Whilst holding the sample under constant strain, the sample was then heated up to $180{ }^{\circ} \mathrm{C}$, i.e., $\sim 40{ }^{\circ} \mathrm{C}$ below its melting point. Upon reaching $180{ }^{\circ} \mathrm{C}$, the polymer had softened, and the measured force exerted on the sample to maintain a constant strain had dropped to between $30-40 \mathrm{~N}$. Once the sample was at $180{ }^{\circ} \mathrm{C}$, it was annealed at this temperature for 4 hours maintaining a constant force. After the annealing period the sample was cooled back to room temperature under constant strain.

\section{X-ray fiber diffraction measurements}

X-ray fiber diffraction experiments were carried out on the Macromolecular crystallography and BioSAXS beamline F1 at Cornell High Energy Synchrotron Source (CHESS). The experiments were run with a beam energy of $12.686 \mathrm{keV}$, equivalent to a X-ray wavelength of $0.9773 \AA$ A. A Quantum-270 area detector was used to collect diffraction patterns that are stored in 16-bit image files. The sample-todetector distance was $145 \mathrm{~mm}$, which was calibrated using silver behenate. The X-ray exposure time for each image was $15 \mathrm{~s}$. The sample was mounted as shown schematically in Figure 2a. The stretched polymer sample was mounted on a frame that was itself attached to a magnetic coupling. This entire piece was attached to a rotatable spindle, so that the tilting angle between the plane of the sample surface and the incident X-ray beam could be adjusted. To ensure correct rotation, the front surface of the polymer film was positioned to intersect the axis of the spindle rotation. The spindle rotation axis was also perpendicular to the incident $\mathrm{X}$-ray direction. Figure $2 \mathrm{~b}$ uses the $(00 l)$ reflection nodes in reciprocal space to demonstrate the scattering geometry. Since the incident X-ray beam direction was initially perpendicular to the fiber axis, to precisely measure the peak position in the meridian direction, the sample needs to be rotated around the spindle axis, so that $(00 l)$ nodes could intersect with the Ewald sphere. The tilting angle in this intersecting geometry is denoted as $\delta$. After projection (see Fig. 2b), the position of diffraction node on the flat detector, $x$, is related to scattering angle $2 \theta$ by:

$$
\tan 2 \theta=x / R
$$

and $R$ is the sample-to-detector distance. To determine the angle of the intersecting geometry, $\delta$, the sample was rotated around the spindle axis and diffraction patterns were collected at steps of $1^{\circ}$. The integrated intensity of a given $(00 l)$ reflection node as a function of tilt angle was then plotted, and the angle at which this intensity reached a maximum corresponds to $\delta$. Diffraction patterns shown in this 
paper were collected at $\delta=0^{\circ}$. More patterns at different tilt angles are shown in the Supplementary Material.

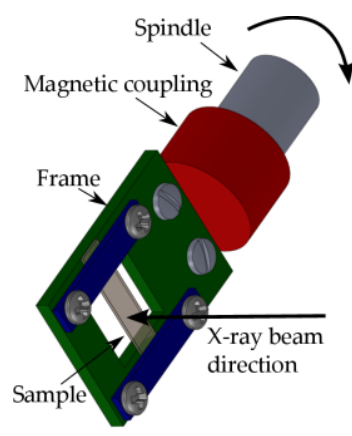

(a)

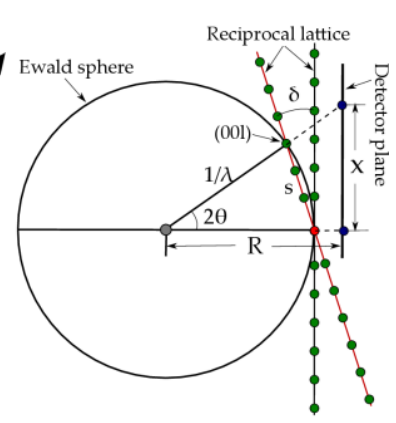

(b)

Figure 2. (a) Schematic diagram of the sample mount and spindle rotation used in the fiber diffraction experiments. (b) Ewald construction of diffraction geometry together with definition of scattering geometry (further details in the text).

\section{Molecular modeling}

Accelrys Materials Studio software was used to construct models of PEF polymer chains in a molecular crystal. The molecular packing within the unit cell and subsequent energy minimization was performed using the Forcite module, utilizing the COMPASS force field. The atomic fractional coordinates were generated after geometry/energy minimization of crystal structures, from which simulated 2D fiber diffraction patterns were generated as described below.

\section{Fiber diffraction pattern simulation}

With knowledge of unit cell parameters, atomic positions (in one unit cell), and the polymer chain alignment direction, 2D fiber diffraction patterns can be simulated. The starting point is to determine the structure factor, $F_{h k l}$ by considering all fractional coordinates of the atoms in a unit cell, $\left(p_{j}, q_{j}, r_{j}\right)$, and the corresponding atomic scattering factor, $f_{j}$, using the following equation:

$$
F_{h k l}=\sum_{j=1}^{n} f_{j} \exp \left[-i 2 \pi\left(p_{j} h+q_{j} k+r_{j} l\right)\right]
$$

where $(h k l)$ are the Miller indices defining the nodes in reciprocal space. The powder-averaged scattering intensity for a given $(h k l)$ node is given by:

$$
I_{h k l}=\frac{1}{4 \pi s_{h k l}^{2}}\left|F_{h k l}\right|^{2}
$$


where $s_{h k l}=(2 / \lambda) \sin \theta_{h k l}$ is the modulus of the scattering vector of a given $(h k l)$ node, $2 \theta_{h k l}$ is the scattering angle and $\lambda$ is the $\mathrm{X}$-ray wavelength.

In producing a sample for fiber diffraction experiments, polymer chains were aligned along the (uniaxial) strain direction, i.e., the fiber axis, and crystals within the sample therefore possess fiber symmetry around the fiber axis. In such cases it is often more convenient to use alternative orthogonal coordinates to define reciprocal space, as shown in Figure 3, where $\mathbf{s}_{1}, \mathbf{s}_{2}$ and $\mathbf{s}_{3}$ are three mutually orthogonal axes. Following convention, the $\mathbf{s}_{3}$ axis is taken as the fiber axis. ${ }^{13,14}$ It should be pointed out that the $\mathbf{c}^{*}$-axis in reciprocal space is only aligned with $\mathbf{s}_{3}$ when the $c$-axis is perpendicular to the $a b$-plane in real space.

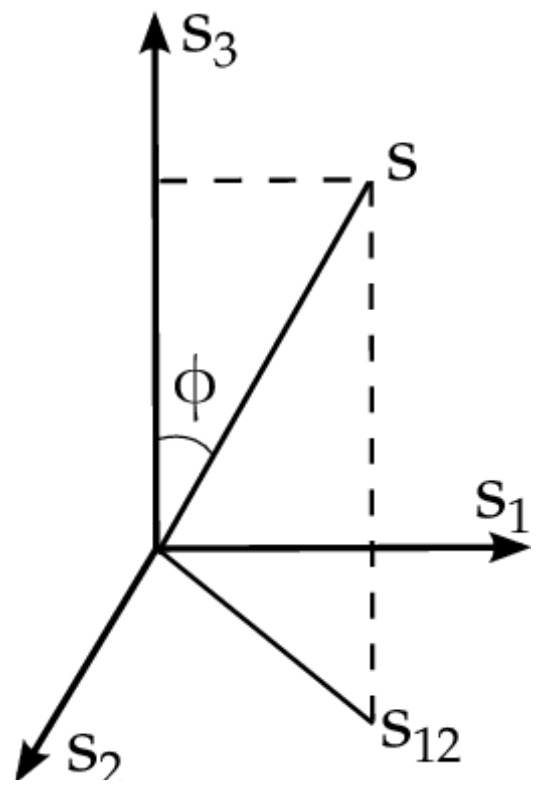

Figure 3. Schematics of orthogonal coordinate system in reciprocal space. For sample possessing fiber symmetry, reciprocal lattice can be defined using modulus of scattering vector $s$ and polar angle $\phi$.

For systems possessing fiber symmetry, with the aforementioned crystal orientation in the $\mathbf{s}_{3}$ direction, the scattering intensity is only dependent on $\boldsymbol{s}_{12}=\sqrt{\boldsymbol{s}_{1}^{2}+\boldsymbol{s}_{2}^{2}}$ and $\mathbf{s}_{3}$, or, equivalently, on $\boldsymbol{s}$ and $\phi$, where $\boldsymbol{s}=\sqrt{\boldsymbol{s}_{12}^{2}+\boldsymbol{s}_{3}^{2}}$, and $\phi$, is the polar angle, defined as the angle between $\mathbf{s}_{3}$ and $\boldsymbol{s}$. Therefore, a $2 \mathrm{D}$ fiber diffraction pattern contains all the information required to describe the reciprocal lattice. For crystalline samples, scattering intensities are localized in the vicinity of each $(h k l)$ node, and the anisotropic scattering intensity distribution, $J(s, \phi)$, can be approximated as a multiplication of the powder-averaged intensity (Eq. 3) and a kernel defining the orientation condition, $F\left(\phi, \phi_{h k l}\right):^{15,16}$

$$
J(s, \phi)=I_{h k l}\left(s_{h k l}\right) F\left(\phi, \phi_{h k l}\right)
$$


The $F\left(\phi, \phi_{h k l}\right)$ kernel is obtained from a Fourier transform of the (real space) orientation distribution function (ODF), $g(\beta)$, where $\beta$ is the so-called misorientation angle. There are only limited forms of $g(\beta)$ that yield explicit analytic expressions for the $F\left(\phi, \phi_{h k l}\right)$ kernel, and the Onsager function is one which is appropriate for the current work, defined as follows: ${ }^{15}$

$$
g(\beta)=p \cosh (p \cos \beta) / \sinh p
$$

where $p$ is a parameter determining the breadth of orientation distribution. By adopting the Onsager function form for the ODF the $F\left(\phi, \phi_{h k l}\right)$ kernel can then be simplified to the analytic form written as:

$$
F\left(\phi, \phi_{h k l}\right)=\frac{p}{\sin (p)} \cosh \left(p \cos \phi \cos \phi_{h k l}\right) I_{0}[n]
$$

where $I_{0}[n]$ is the modified Bessel function of the first kind of order zero, and $n=p \sin \phi \sin \phi_{h k l}$.

\section{Results and Discussion}

Part of the fiber diffraction pattern of the PEF sample, collected at zero tilt angle, is shown in Figure 4a. Even without tilting, and thereby optimizing for the angle $\delta$, a series of meridian diffraction arcs, rather than well-defined diffraction spots, were clearly visible in the diffraction pattern. In principle, when the $\mathbf{c}^{*}$-axis is parallel to the $\mathbf{s}_{\mathbf{3}}$ direction, diffraction spots should be invisible, because they should not be able to intersect with the Ewald sphere at zero tilt angle (as shown in Figure 2b). However, in reality diffraction intensity is observed for one of two reasons. Firstly, various broadening effects such as small crystal size or disorder induce smearing of the diffraction spots in reciprocal space. An effect known as smearing. The second reason could result if the $\mathbf{c}^{*}$-axis had a small oblique angle with respect to $\mathbf{s}_{\mathbf{3}}$ (as may be seen in triclinic cells for example). The small oblique angle nevertheless causes diffraction arcs that appear at the meridian after intersecting with the Ewald sphere. An effect known as broadening.

To discriminate between these effects, tilting measurements were performed (see Experimental Method section). By tilting the sample in an increment of $1^{\circ}$, the intensity of all meridian arcs first increased, reached a maximum, and then decreased. This verified that the meridian arcs were caused by smearing rather than broadening, since no maxima would be seen in the latter case. These tilt-measurements also confirm that the $\mathbf{c}^{*}$-axis is in the same direction as the $\mathbf{s}_{\mathbf{3}}$ axis. If $\mathbf{c}^{*}$ were not aligned with the fiber axis, a given $(h k l)$ node in the $l$-th layer in reciprocal space would produce a circle. In the fiber tilting experiment, the spots on the circle would gradually intersect with Ewald sphere, producing two diffraction spots for any $(h k l)$ plane that would become increasingly separated with increasing tilt angle (see Figure S4 in the supplementary information). However, this behavior was not observed in our experiment. By contrast only single localized diffraction spots corresponding to $(00 l)$ planes were visible, 
the intensity of which change as a function of tilting angle were consistent, similar to what is shown in Fig $4 \mathrm{~b}$ for the (008) plane (further diffraction patterns at different tilting angles can be found in the supplementary material). This confirmed that the meridian diffraction spots were not due to existence of an oblique angle between $\mathbf{c}^{*}$ and the fiber axis, and indeed confirm that both $\mathrm{c}^{*}$ is parallel to the fiber axis.

The integrated intensity of a meridian arc, in this case from the (008) reflection, as a function of tilt angle is shown in Figure $4 \mathrm{~b}$. The position of intensity maximum corresponds to the $\delta$-angle for a full intersection of (008) plane to the Ewald sphere, from which the repeating unit length $c$, can be precisely determined (given that $c=1 / s$ ). The same procedure was also applied to the (002) and (004) reflections. The averaged repeating unit length from these three calculations gave $c=20.296 \AA$. A unit cell $c$-axis dimension of $20.296 \AA$ is much larger than the length of one PEF monomer, but based on dimensional analysis it is a length scale consistent with the inclusion of two monomers in the unit cell. This value of $c$ was used in the subsequent structure determination.
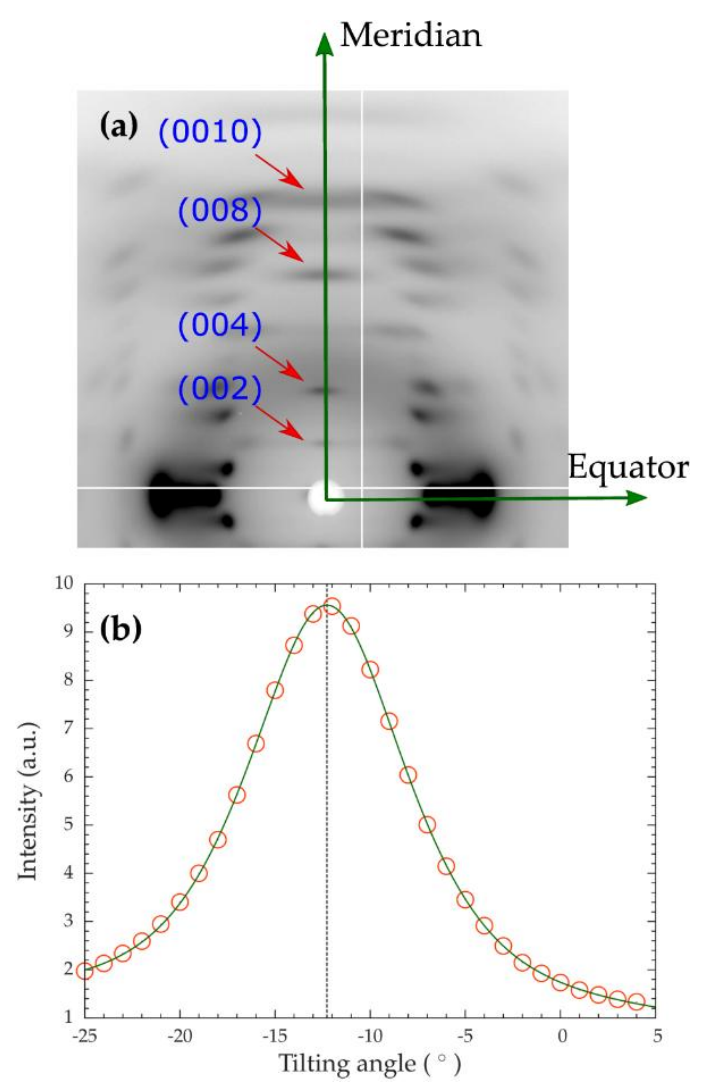

Figure 4. A portion of the diffraction pattern of PEF collected at zero tilting angle (a), with a representative example of change of integrated scattering intensity as a function of tilting angle in this case of the (008) reflection (b). 
Since $\mathbf{c}^{*}$-axis is in the same direction as $\mathbf{s}_{\mathbf{3}}$, orthorhombic and monoclinic unit cells with the first setting (i.e. where the crystallographic axis $c$ is the unique axis, and the $a$-and $b$-axis are orthogonal to it) are two reasonable options for the crystal structure of the polymer. Positions of some diffraction spots with small $\boldsymbol{s}$-values were used to determine the remaining cell parameters, namely, $a, b$, and the oblique angle, $\gamma$ (for monoclinic cell). Comparisons to experimental data show that a monoclinic cell with $a=5.784 \AA$, $b=$ $6.780 \AA, c=20.296 \AA$, and $\gamma=103.3^{\circ}$ (first setting) was able to index all the experimental diffraction spots. The calculated density of the crystalline PEF based on these parameters is $1.562 \mathrm{~g} / \mathrm{cm}^{3}$. As a comparison, the density of the PEF in its fully amorphous state, as measured using a density gradient column is $1.438 \mathrm{~g} / \mathrm{cm}^{3}$. It is interesting to note that although our unit cell parameters are different from those derived by Kazaryan and Medvedeva, ${ }^{12}$ both models give the same crystal density. Our unit cell containing two chains has a volume equal to $774.6 \AA^{3}$, which is twice as large as compared to Kazaryan and Medvedeva's triclinic cell volume (388 $\left.\AA^{3}\right)$. 


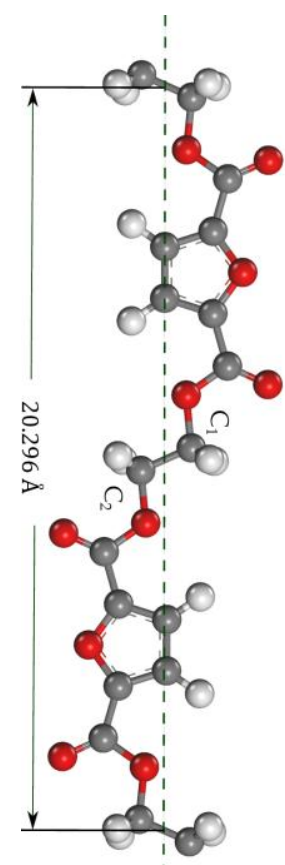

(a)

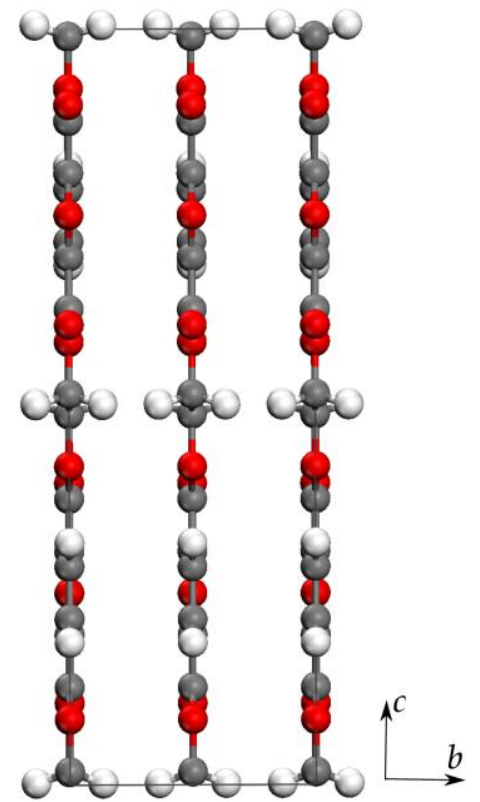

(b)

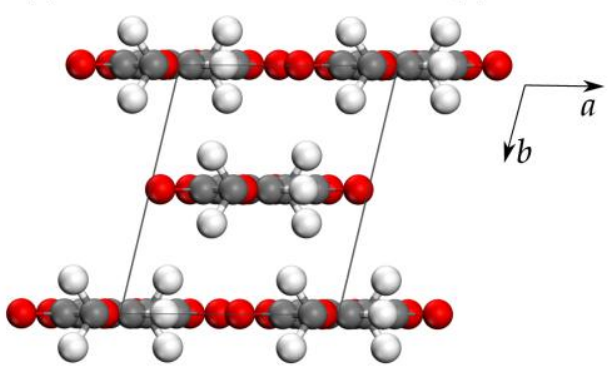

(c)

Figure 5. Chain conformation of PEF in one repeating unit (a); possible chain packing in a monoclinic unit cell (see details in the text), seen through [100] direction (b); chain packing seen through [001] direction $(\mathbf{c})$.

On detailed examination, the XRFD pattern (see Fig. 4a) reveals the absence of reflections from ( $00 l$ ) planes when $l$ is equal to an odd number, which suggests the existence of a $2_{1}$ screw-axis. Taking this factor and unit cell dimensions into account, a possible structure of PEF chain packing in the unit cell was derived using molecular modeling, as shown in Fig. 5. The polymer chains in the model have a transplanar conformation in the unit cell, so that the $2_{1}$ axis goes through the mid-point of the alkane bond between $\mathrm{C}_{1}$ and $\mathrm{C}_{2}$, as shown in Figure $5 a$. The monoclinic cell can accommodate two chains, with one chain located at the edge of the unit cell and the other chain in the center of the $a b$-plane. After energy minimization, the resulting monoclinic unit cell structure (as shown in Figure 5) gave a $c$-axis length of $20.233 \AA$, which is close to the experimentally determined value. From a geometric point of view, the second chain can also be allowed to go through the mid-point of the $b$-axis. However, when doing this the calculated diffraction pattern, did not match well with experimental data, and for example, the (010) and 
(100) reflections are not absent as should be case, confirming that the former chain arrangement is more appropriate.

Assuming therefore that the second chain must pass through the center of the $a b$-plane, we can further refine the model structure. Intuitively, simply putting only one PEF monomer in the asymmetric unit as a start point, and by applying symmetry operations in space group $\mathrm{P} 2{ }_{1} / \mathrm{n}$ (No. 13 in International Table of Crystallography) ${ }^{17}$, a total of four monomers with the correct monomer connectivity (bonding) can be included in the unit cell, as shown in Figure 5b and 5c. However, a problem arises when generating the unit cell using these symmetry operations as the simulated diffraction pattern did not exactly match the experimental results with respect of the weak (006) reflection. The (006) peak was not discernible in experimental diffraction patterns collected at all tilt angles. This effect is not due to extinction rules of all space groups for a monoclinic cell, and must be attributed to details of chain packing.

A likely explanation of the weak (006) peak is the effect of inter-chain interference. Consider a simple case when the center-chain is translated along the $c$-axis by a distance $\Delta r$ (in fractional units), with respect to the edge-chain, in this case the interference function for the $(00 l)$ planes is given by $1+$ $\cos 2 \pi \Delta r l .{ }^{18}$ Setting this term to zero yields $\Delta r=1 / 12,3 / 12,5 / 12$, etc. Packing energies for unit cells with these first three center-chain-staggering structures in the series, determined from the molecular modeling, are $13.68 \mathrm{Kcal} / \mathrm{mol}, 17.15 \mathrm{Kcal} / \mathrm{mol}, 16.82 \mathrm{Kcal} / \mathrm{mol}$, respectively, whilst the packing energy for a unit cell consisting of two chains without staggering was found to be considerably higher at 46.54 $\mathrm{Kcal} / \mathrm{mol}$. The lower packing energy for staggered chains can be rationalized because this arrangement avoids the geometric hindrance of the hydrogen atoms in the central $\mathrm{C}_{1}-\mathrm{C}_{2}$ alkyl group, as seen in Figures $5 b$ and $5 c$.

The implication of center-chain-staggering means that all other symmetry elements except the $2_{1}$ axis are not possible. Therefore, the most likely space group that explains this behavior is $\mathrm{P} 2{ }_{1}$. In this case, the asymmetric unit consists of two monomers, one located at a unit cell edge, and the other at the cell center, and the other 2 monomers are generated by a $2_{1}$ symmetry operation. This type of structure is not completely unknown and resembles that of cellulose-I and cellulose-II crystals, which are composed of 2 chains with a staggered central chain. ${ }^{19-21}$

Since unit cells with $1 / 12,3 / 12$, and 5/12-staggering of center chain have very similar packing energies, a diffraction pattern was simulated based on an equal mixture of these three individual cells. This is called register disorder, and the diffraction pattern simulation method is equivalent to the treatment of substitution disorder frequently met in metal alloys, ${ }^{18}$ but has also been found in some other rigid-chain polymers such as poly(paraphenylene vinylene) and poly(p-phenylene benzobisoxazole). ${ }^{22,23}$ Figure 6 
shows results of the experimental pattern compared with that calculated from the model unit cell assuming a mixed cell containing equal amounts of 1/12,3/12, and 5/12-staggered chains. The simulated pattern was distortion corrected and re-mapped onto a matrix (using an inverse Fraser correction) so that it can be directly compared with the experimental pattern. ${ }^{13,14,16,24-26}$ The upper-left and lower-right quadrants in Figure 6 are the experimental results, with the simulated patterns shown in the upper-right and lower-left quadrants. This register disordered model now properly accounts for the extinction of the (006) peak. However, although most of the simulated diffraction spots match exceptionally well with the experimental data, the (019) plane was unaccountable absent in the simulated pattern (as indicated by the arrow in Fig. 6).

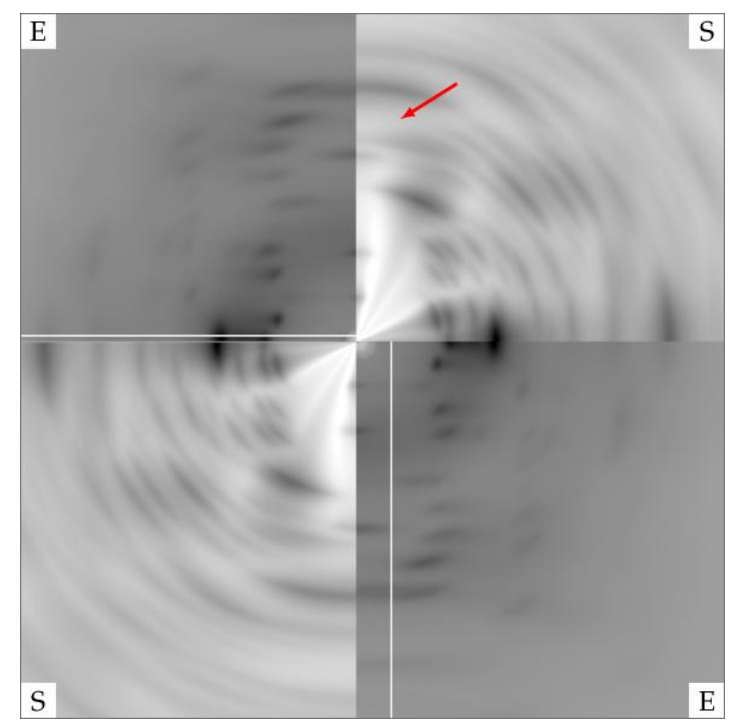

Figure 6. Comparison of experimental fiber diffraction pattern (upper-left and lower-right) and simulated diffraction pattern using a mixed cell consisting of 1/12,3/12, and 5/12 center-chain staggering. Letters $\mathbf{E}$ and $\mathbf{S}$, stand for 'experimental' and 'simulated', respectively. The tilting angle is zero degree. Scattering intensity is displayed on a logarithmic scale. The red arrow indicates the expected position (and therefore absence) of the (019) reflection in the simulated diffraction pattern.

In order to account for the discrepancy in the (019) reflection, energy minimization of molecular models was performed for packing schemes with different center-chain-staggering. In performing these energy minimizations the unit cell dimensions were fixed, but the chains were allowed to undergo local motions, such as rotation and translation, whilst maintaining the $\mathrm{P} 2_{1}$ space group. After minimization, packing energies of $2.54 \mathrm{Kcal} / \mathrm{mol}, 7.25 \mathrm{Kcal} / \mathrm{mol}$, and $7.18 \mathrm{Kcal} / \mathrm{mol}$ were determined for the $1 / 12-, 3 / 12-$, and 5/12-staggered structures, respectively. Fiber diffraction patterns were subsequently simulated based on the new coordinates of each individual cell and on mixtures of these cells following the register disorder 
approach. An exact match to the experimental data was found when using a mixture of 3/12- and 5/12staggering (see Figure 7), which was able to account for the systematic occurrences and absences of reflections, including the extinction of the (006) peak and the presence of (019) peak. As seen in Figure 7, the result of including these staggering in the energy minimized PEF chains is to induce slight bending of the chains. The monomer is off-axis by $\sim 3^{\circ}$ and $\sim 7^{\circ}$ in cells containing 3/12 and 5/12-staggered chains, respectively. Similar bent chain conformations are not unknown and have also been observed in the unit cell structures for some other polymers including poly(tetramethylene terephthalate), poly(pphenylenebenzobisthiazole) and poly(p-phenylenebenzobisoxazole). ${ }^{27,28}$

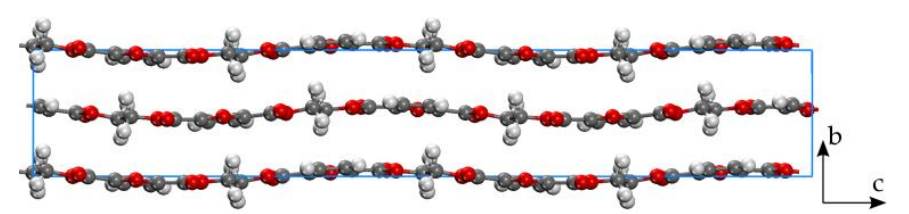

(a)

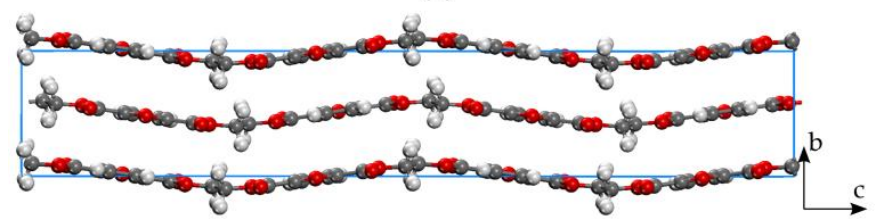

(b)

Figure 7. Packing of PEF chains after performing energy minimization. Center-chain is staggered by $3 / 12$ (a) and 5/12 (b), with respect to the edge-chains. Polymer chains are slightly bended.

A comparison of experimental results and the simulated diffraction pattern using 3/12 and 5/12 centerchain-staggering structure is shown in Figure 8. Data presentation is the same as what is shown in Figure 6 , with the most obvious diffraction spots indexed. A more complete list of reflection planes, including peak positions from the simulated pattern and observed values from experiment, and a qualitative classification of peak intensity is given in the supporting material. As shown in Figure 8, all experimental diffraction peaks were successfully reproduced by the simulated data confirming the unit cell structure. However, it is difficult to quantitatively compare the integrated intensity of the diffraction spots between the experimental and simulated data. The reason for this is that in the experimental data severe peak overlapping was observed due to small crystal sizes in the sample, coupled with large disorder effects and low degree of crystallinity. Unlike the diffraction pattern, which is shown on a logarithmic scale, the scattering intensity profile of each layer line often consisted of several broad intensity maxima, which introduces ambiguity in determination of peak position and integrated intensity. Nevertheless, the 
proposed crystal structure successfully explains the appearance and absence of all the diffraction spots in experimental results. Additional detail about the crystal structure will require fiber diffraction patterns of even higher quality, which is obviously a challenging task given how poorly PEF crystallizes.

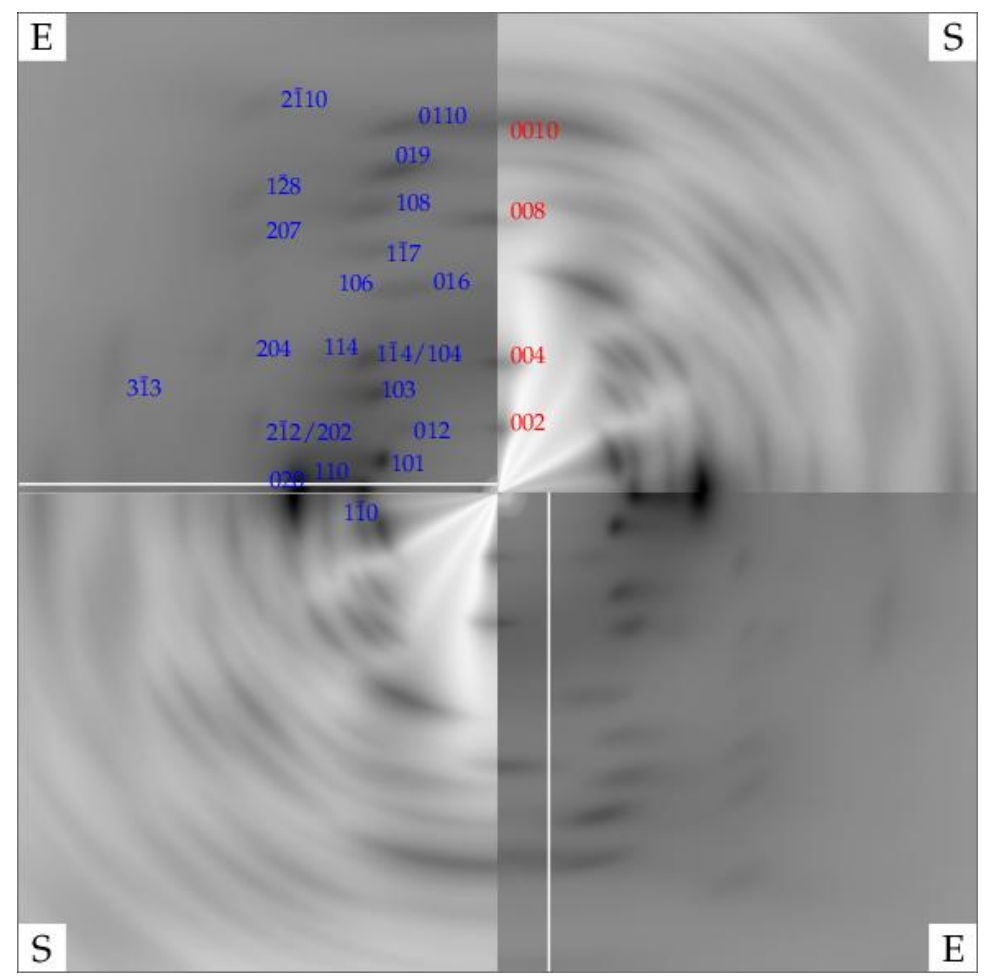

Figure 8. Comparison of experimental fiber diffraction (upper-left and lower-right) and simulated pattern (upper-right and lower-left). Simulated pattern was computed using a mix cell consisting of $3 / 12$ and 5/12 center-chain staggering, after energy minimization. Major diffraction spots are indexed. Scattering intensity is displayed in logarithmic scale.

\section{Conclusion}

By combining fiber diffraction pattern simulations and molecular modeling, the crystal structure of PEF was determined. A monoclinic unit cell with a space group of $\mathrm{P} 2_{1}$ and cell parameters of $a=5.784 \AA$, $b=6.780 \AA, c=20.296 \AA$, and $\gamma=103.3^{\circ}$ (first setting) was able to index all observed diffraction spots. The density of this crystalline structure is $1.562 \mathrm{~g} / \mathrm{cm}^{3}$. The $c$-axis length indicated that the PEF monoclinic unit cell consisted of two monomers. Energy minimization on the model structure of the unit cell were undertaken and simulated 2D fiber diffraction patterns derived. These were compared to the experimental data and it was found to properly account for the diffraction pattern it was necessary to include staggering of the center-chain of the unit cell with respect to the edge-chain. PEF chains were 
shown to adopt a slightly bent conformation in the unit cell, consisting of a mixture of both 3/12- and 5/12-staggered-chains.

\section{Acknowledgement}

We gratefully acknowledge funding for this work from the Coke Cola Company. The crystallographic data is based upon measurements conducted at the Cornell High Energy Synchrotron Source (CHESS), which is supported by the National Science Foundation and the National Institutes of Health/National Institute of General Medical Sciences under NSF award DMR-1332208, using the Macromolecular Diffraction at CHESS (MacCHESS) facility, which is supported by award GM-103485 from the National Institutes of Health, through its National Institute of General Medical Sciences.

\section{Reference}

1. Burgess, S. K., Lee, J. S., Mubarak, C. R., Kriegel, R. M. \& Koros, W. J. Caffeine antiplasticization of amorphous poly (ethylene terephthalate): Effects on gas transport, thermal, and mechanical properties. Polymer 65, 34-44 (2015).

2. Burgess, S. K. et al. Chain mobility, thermal, and mechanical properties of poly (ethylene furanoate) compared to poly (ethylene terephthalate). Macromolecules 47, 1383-1391 (2014).

3. Burgess, S. K. et al. Water sorption in poly (ethylene furanoate) compared to poly (ethylene terephthalate). Part 1: Equilibrium sorption. Polymer 55, 6861-6869 (2014).

4. Burgess, S. K. et al. Water sorption in poly (ethylene furanoate) compared to poly (ethylene terephthalate). Part 2: Kinetic sorption. Polymer 55, 6870-6882 (2014).

5. Burgess, S. K., Karvan, O., Johnson, J., Kriegel, R. M. \& Koros, W. J. Oxygen sorption and transport in amorphous poly (ethylene furanoate). Polymer 55, 4748-4756 (2014).

6. Burgess, S. K., Mubarak, C. R., Kriegel, R. M. \& Koros, W. J. Physical aging in amorphous poly (ethylene furanoate): Enthalpic recovery, density, and oxygen transport considerations. J. Polym. Sci. Part B Polym. Phys. 53, 389-399 (2015).

7. Burgess, S. K., Kriegel, R. M. \& Koros, W. J. Carbon Dioxide Sorption and Transport in Amorphous Poly (ethylene furanoate). Macromolecules 48, 2184-2193 (2015). 
8. Daubeny, R. de P. \& Bunn, C. The crystal structure of polyethylene terephthalate. in Proceedings of the Royal Society of London A: Mathematical, Physical and Engineering Sciences 226, 531-542 (The Royal Society, 1954).

9. Stoclet, G., du Sart, G. G., Yeniad, B., de Vos, S. \& Lefebvre, J. Isothermal crystallization and structural characterization of poly (ethylene-2, 5-furanoate). Polymer 72, 165-176 (2015).

10. van Berkel, J. G. et al. Isothermal Crystallization Kinetics of Poly (Ethylene 2, 5-

Furandicarboxylate). Macromol. Mater. Eng. 300, 466-474 (2015).

11. Tsanaktsis, V., Papageorgiou, D. G., Exarhopoulos, S., Bikiaris, D. N. \& Papageorgiou, G. Z. Crystallization and Polymorphism of Poly (ethylene furanoate). Cryst. Growth Des. 15, 5505-5512 (2015).

12. Kazaryan, L. \& Medvedeva, F. X-Ray study of the structure of the polyester of furan-2, 5dicarboxylic acid. Vysok. Soedin Ser B 10, (1968).

13. Stribeck, N. On the determination of fiber tilt angles in fiber diffraction. Acta Crystallogr. A 65 , 46-47 (2009).

14. Stribeck, N. \& Nöchel, U. Direct mapping of fiber diffraction patterns into reciprocal space. J. Appl. Crystallogr. 42, 295-301 (2009).

15. Burger, C., Hsiao, B. S. \& Chu, B. Preferred orientation in polymer fiber scattering. J. Macromol. Sci. Part C Polym. Rev. 50, 91-111 (2010).

16. Mao, Y. et al. Wide-angle X-ray scattering study on shear-induced crystallization of propylene-1butylene random copolymer: Experiment and diffraction pattern simulation. Macromolecules 44, 558565 (2011).

17. Hahn, T. \& Crystallography, I. U. of. Space-group Symmetry. (International Union of Crystallography, 2006).

18. Guinier, A. X-ray Diffraction in Crystals, Imperfect Crystals, and Amorphous Bodies. (Dover, 1994). 
19. Langan, P., Nishiyama, Y. \& Chanzy, H. A revised structure and hydrogen-bonding system in cellulose II from a neutron fiber diffraction analysis. J. Am. Chem. Soc. 121, 9940-9946 (1999).

20. Langan, P., Nishiyama, Y. \& Chanzy, H. X-ray structure of mercerized cellulose II at $1 \backslash$ AA resolution. Biomacromolecules 2, 410-416 (2001).

21. Nishiyama, Y., Langan, P. \& Chanzy, H. Crystal structure and hydrogen-bonding system in cellulose I $\beta$ from synchrotron X-ray and neutron fiber diffraction. J. Am. Chem. Soc. 124, 9074-9082 (2002).

22. Granier, T., Thomas, E. L. \& Karasz, F. E. Paracrystalline structure of poly (paraphenylene vinylene). J. Polym. Sci. Part B Polym. Phys. 27, 469-487 (1989).

23. Tashiro, K. et al. Confirmation of the crystal structure of poly (p-phenylene benzobisoxazole) by the X-ray structure analysis of model compounds and the energy calculation. J. Polym. Sci. Part B Polym. Phys. 39, 1296-1311 (2001).

24. Fraser, R., Macrae, T., Miller, A. \& Rowlands, R. d. Digital processing of fibre diffraction patterns. J. Appl. Crystallogr. 9, 81-94 (1976).

25. Mao, Y. et al. Time-Resolved Synchrotron X-ray Scattering Study on Propylene-1-Butylene Random Copolymer Subjected to Uniaxial Stretching at High Temperatures. Macromolecules 45, 951$961(2011)$.

26. Mao, Y., Li, X., Burger, C., Hsiao, B. S. \& Tsou, A. H. 2D WAXS/SAXS study on isotactic propylene-1-butylene random copolymer subjected to uniaxial stretching: The influence of temperature. Polymer 54, 1432-1439 (2013).

27. Hall, I. \& Pass, M. Chain conformation of poly (tetramethylene terephthalate) and its change with strain. Polymer 17, 807-816 (1976).

28. Fratini, A. V., Lenhert, P. G., Resch, T. J. \& Adams, W. W. Molecular Packing and Crystalline Order in Polybenzobisoxazole and Polybenzobisthiazole Fibers. MRS Proceedings, 134, 431-446 (1988) 


\section{TOC Graphics}

Comparison of experimental fiber diffraction (upper-left and lower-right) and simulated pattern (upperright and lower-left) of Poly(ethylene furanoate).

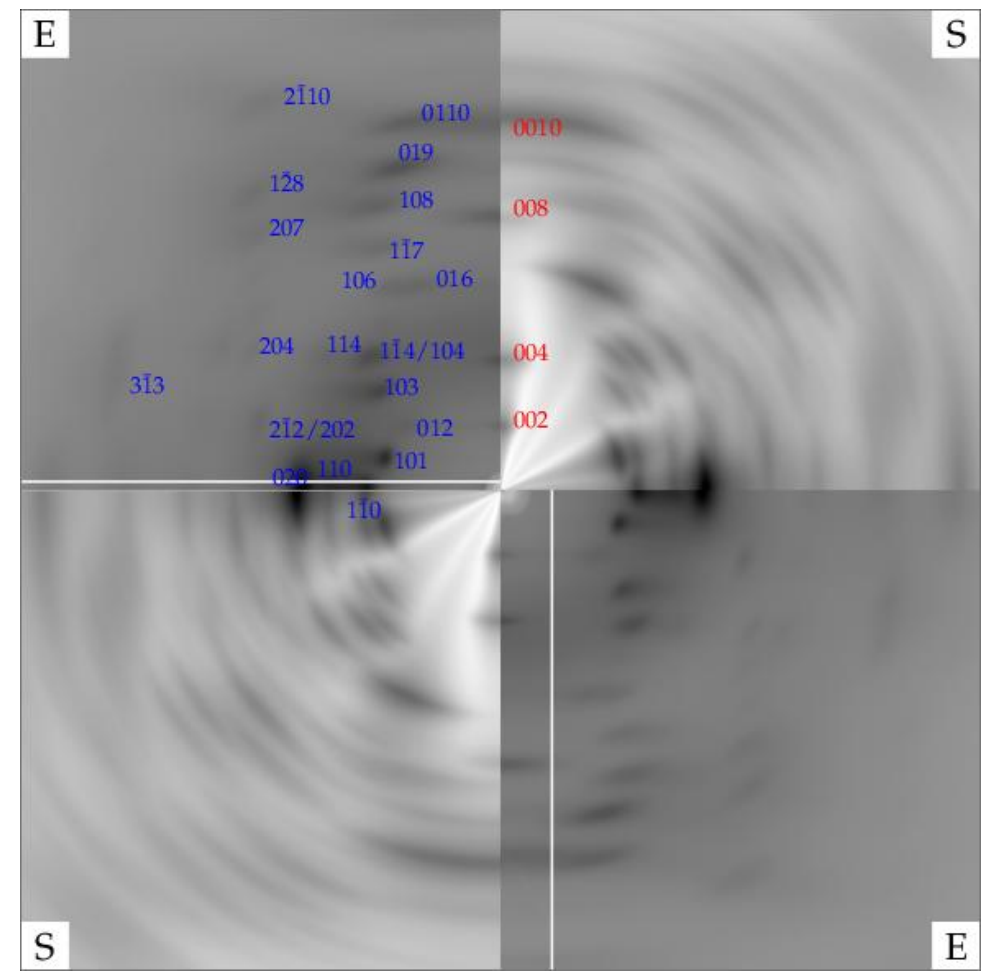

